\title{
Efficacy of Impression (Tricyclazole 45\%+Hexaconazole 10\%WG) against Fruit rot (Colletotrichum sp.) and Leaf spot (Alternaria and Cercospora) of Pomegranate under field condition
}

\author{
Suresh D. Ekabote ${ }^{1 *}$, Pruthviraj $^{2}$, Divyajyothi, $\mathrm{U}^{3}$ and Nalina, S. O. ${ }^{4}$ \\ ${ }^{\prime}$ Department of Horticulture Crop Protection, College of Horticulture, Hiriyur, UAHS, Shivamogga \\ ${ }^{2}$ Department of Plant Pathology, College of Agriculture, Shivamogga, UAHS, Shivamogga \\ ${ }^{3} \mathrm{ZBNF}, \mathrm{ZAHRS}$, Babbur farm, Hiriyur \\ ${ }^{4}$ Department of Horticulture Crop Protection, College of Horticulture, Hiriyur \\ "CorrespondingauthorEmail: sureshde@uahs.edu.in
}

(Submitted on June 26, 2020; Accepted on November 27, 2020)

\section{ABSTRACT}

\begin{abstract}
The pomegranate (Punica granatum L.), Is one of the ancient and highly priced favorite fruit which belongs to family Lythraceae of order Myrtales and it is mainly grown in tropical and subtropical regions of the world. In recent years, successful cultivation of pomegranate is faced by many constraints, among them, pest and diseases are the major ones. Common diseases of pomegranate leaf and fruits include Anthracnose and Cercospora and Alternaria leaf spot and these diseases cause severe loss to pomegranate crop. Therefore, a field experiment was carried out on the effect of Tricyclazole 45\%+Hexaconazole $10 \% \mathrm{WG}$ against leaf spots and fruit rot of pomegranate during 2018-19 and 2019-2020 at College of Horticulture, Hiriyur. Experimental results revealed that all the treatments significantly reduced the leaf spots and fruit rot disease severity over untreated control. Tricyclazole 45\%+Hexaconazole $10 \%$ WG@ $1.25 \mathrm{gm} / \mathrm{L}$ was most effective in the management of leaf spots $(9.64$ and 12.00 PDI) and anthracnose of pomegranate (7.17 and 12.87 PDI), respectively followed by the same fungicide $a 1.00 \mathrm{gm} / \mathrm{L}$ with a yield of 5.24 tons/ha and 5.64 tons/ha, respectively

Keywords: Cercospora and Alternaria leaf spot, Anthracnose, Tricyclazole + Hexaconazole
\end{abstract}

\section{INTRODUCTION}

Pomegranate (Punica granatum L.) is the most important horticultural crop of Chitradurga district coming under southern dry zone of Karnataka. Pomegranate suffers from various diseases among these leaf/fruit spot caused by various organisms such as, Colletotrichum gloeosporioides, Cercospora punicae, Alternaria alternata, Sphaceloma punicae, Drechslera sp., and Phomopsis sp., take a heavy toll on the crop (Jamadar and Patil, 2007). This results in drastic reduction in the yield as well as ultimate marketability by way of severe spotting of the produce. There are several conventional fungicides being used by the farmers with no avail. Hence there is a need to explore new chemical formulations, which are highly efficient in managing these diseases effectively. Hence an attempt was made to identify the performance of the new molecules against the leaf spots and fruit rot of pomegranate.

\section{MATERIAL AND METHODS}

Field experiment was conducted in two Kharif cropping seasons of 2018-19 and 2019-20 on the management of anthracnose (Colletotrichum gloeosporioides), Cercospora and Alternaria leaf spot (Cercospora punicae and Alternaria spp.) affecting pomegranate crop at College of Horticulture, Hiriyur, UAHS, Shivamogga, Karnataka. The soil of the experimental field was red sandy loam which was acidic to neutral $(5-7 \mathrm{pH})$ in reaction. The experiment was carried out for 2 years on a fixed plot where pomegranate orchard was established. Different fungicides with varied mode of action having different degrees of systemic movement and wide spectrum mode of action against different taxonomic group of fungi were used for the study (Bartlett et al., 2002; Nithyameenakshi et al., 2006; De and Mandal, 2007). The experiment was laid out with Randomized Block Design (RBD). The experiment consists of 10 treatments including control and were laid out in plots with size $8 \mathrm{~m}$ X $6 \mathrm{~m}\left(48 \mathrm{~m}^{2}\right)$ with spacing $12 \mathrm{ft}$ X $10 \mathrm{ft}$ and the variety used was Super Bhagwa. The treatment fungicides were applied to the Pomegranate field at the beginning of the disease appearance. Spray schedule was repeated at 15 days interval. The observation of incidence of Leaf spots, Fruit spots and Anthracnose diseases was assessed by using the 1-9 score chart and the per cent disease index (PDI) was calculated for each spray as under.

The per cent disease index (PDI) was calculated by the following formula which was given by Wheeler, 1969 and Fruit yield per plant wise were recorded

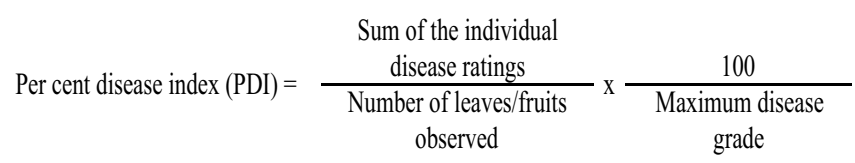

\begin{tabular}{|c|l|c|}
\hline \multicolumn{2}{|c|}{ Treatment details along with checks: } \\
\hline \multicolumn{2}{|c|}{ Treatments } & $\begin{array}{c}\text { Dosage } \\
\text { (mL or g/ L) }\end{array}$ \\
\hline 1 & $\begin{array}{l}\text { Impression (Tricyclazole } \\
45 \%+\text { Hexaconazole 10\% WG) }\end{array}$ & 0.75 \\
\hline 2 & $\begin{array}{l}\text { Impression (Tricyclazole } \\
45 \%+\text { Hexaconazole 10\% WG) }\end{array}$ & 1.00 \\
\hline 3 & $\begin{array}{l}\text { Impression (Tricyclazole } \\
45 \%+\text { Hexaconazole 10\% WG) }\end{array}$ & 1.25 \\
\hline 4 & Hexaconazole 5\% EC & 2.00 \\
\hline 5 & Hexaconazole 5\% EC & 2.50 \\
\hline 6 & Tricyclazole 75\% WP & 0.60 \\
\hline 7 & Tricyclazole 75\% WP & 0.75 \\
\hline 8 & Propineb 70\%WP & 3.00 \\
\hline 9 & $\begin{array}{l}\text { Metiram 55\% + Pyraclostrobin } \\
5 \% \text { WG }\end{array}$ & 3.00 \\
\hline 10 & Untreated Control & NA \\
\hline
\end{tabular}

Phytotoxicity on Pomegranate crop: Fungicides evaluated here have label claim and hence phytotoxicity studies were 
undertaken. Phytotoxicity observations were recorded at 0,1 , $3,5,7 \& 10$ days after each spray of different treatments as per established phytotoxicity parameters.

\section{Phytotoxicity}

\begin{tabular}{|c|l|c|}
\hline $\begin{array}{c}\text { Sl. } \\
\text { No }\end{array}$ & \multicolumn{1}{|c|}{ Treatments } & $\begin{array}{c}\text { Formulation } \\
(\mathbf{m L} / \mathrm{g} / \mathrm{L})\end{array}$ \\
\hline 1 & $\begin{array}{l}\text { Impression (Tricyclazole } \\
45 \%+\text { Hexaconazole 10\% WG) }\end{array}$ & $1.0 \mathrm{~g}$ \\
\hline 2 & $\begin{array}{l}\text { Impression (Tricyclazole } \\
45 \%+\text { Hexaconazole 10\% WG) }\end{array}$ & $1.25 \mathrm{~g}$ \\
\hline 3 & Untreated check & $\mathrm{NA}$ \\
\hline 4 & $\begin{array}{l}\text { Impression (Tricyclazole } \\
45 \%+\text { Hexaconazole 10 WG) }\end{array}$ & $2.5 \mathrm{~g}$ \\
\hline 5 & $\begin{array}{l}\text { Impression (Tricyclazole } \\
45 \%+\text { Hexaconazole 10 WG) }\end{array}$ & $5.0 \mathrm{~g}$ \\
\hline
\end{tabular}

Scores for Phytotoxicity

\begin{tabular}{|c|c|c|}
\hline Sr. & Phytotoxicity (\%) & Score \\
\hline 1 & No phytotoxicity & 0 \\
\hline 2 & $0-10$ & 1 \\
\hline 3 & $11-20$ & 2 \\
\hline 4 & $21-30$ & 3 \\
\hline 5 & $31-40$ & 4 \\
\hline 6 & $41-50$ & 5 \\
\hline 7 & $51-60$ & 6 \\
\hline 8 & $61-70$ & 7 \\
\hline 9 & $71-80$ & 8 \\
\hline 10 & $81-90$ & 9 \\
\hline 11 & $91-100$ & 10 \\
\hline
\end{tabular}

Statistical Analysis: The experimental data collected were analyzed statistically for its significance of difference by the normal statistical procedure adopted for randomized block design. Data from the per cent disease index and yield were analyzed by ANOVA. Per cent data were transformed arc sine where necessary. Differences within the means were compared by using Fisher's LSD (Least Significant Difference) test (Walter, 1997). The level of significance used in ' $\mathrm{F}$ ' and ' $\mathrm{T}$ ' test was $\mathrm{P}=0.05$ and $\mathrm{P}=0.01$. Critical differences were calculated wherever ' $F$ ' test was significant. The values per cent disease index was subjected to angular transformation according to the table given by Sundarraj et al., (1974).

\section{RESULTS AND DISCUSSION}

Efficacy of Tricyclazole $45 \%+$ Hexaconazole $10 \% \mathrm{~W}$ Gagainst Fruit rot (Colletotrichum sp.) disease of Pomegranate during 2018-19: The efficacy of the different treatments during three sprays against fruit rot (Colletotrichum sp.) in Pomegranate during 2018-19 is presented in Table-1. Before the spray, the infestation of Fruit rot (Colletotrichum sp.) was uniform in all the treatments and PDI ranged between 0.82 to 1.09 . At 10 days after first application, Impression (Tricyclazole 45\%+Hexaconazole $10 \% \mathrm{WG}$ @ $1.25 \mathrm{gm} / \mathrm{L}$ was found superior in reducing the fruit rot incidence (2.25 PDI) of Pomegranate followed by Metiram 55\% + Pyraclostrobin 5\% WG with PDI of 3.79 . Hexaconazole 5\% EC @ $2.5 \mathrm{~mL} / \mathrm{L}$ recorded PDI of 4.17. Propineb 70\%WP@3.0 gm/L and Hexaconazole 5\% EC @ $2 \mathrm{~mL} / \mathrm{L}$ recorded PDI of 4.25 which was followed by Impression (Tricyclazole 45\%+Hexaconazole 10\%WG)@ $1.00 \mathrm{gm} / \mathrm{L}$ and $0.75 \mathrm{gm} / \mathrm{L}$ with PDI of 4.31 and 4.39 . The plots treated with Tricyclazole@0.75 gm/L and $0.60 \mathrm{gm} / \mathrm{L}$ recorded PDI of 5.01 and 5.25, respectively. However, the untreated check recorded highest fruit rot incidence by showing 11.50 PDI.

After second spray the treatment of Impression (Tricyclazole 45\% + Hexaconazole 10\%WG)@1.25 gm/Lwas found

Table.1: Bio-efficacy evaluation of (Tricyclazole 45\%+Hexaconazole 10\% WG) against Fruit rot (Colletotrichum sp.) disease of Pomegranate during 2018-19

\begin{tabular}{|c|c|c|c|c|c|c|c|c|}
\hline \multirow{2}{*}{$\begin{array}{l}\text { Tr. } \\
\text { No. }\end{array}$} & \multirow[t]{2}{*}{ Treatments } & \multirow{2}{*}{$\begin{array}{c}\text { Dose } \\
\text { Formulation } \\
\text { (g or } \mathrm{mL} / \text { liter) }\end{array}$} & \multirow{2}{*}{$\begin{array}{l}\text { Before } \\
\text { spray }\end{array}$} & \multicolumn{3}{|c|}{ PDI 10 days after } & \multirow{2}{*}{$\begin{array}{l}\text { \% Reduction } \\
\text { over control } \\
\text { after } 3^{\text {rd }} \text { spray }\end{array}$} & \multirow{2}{*}{$\begin{array}{c}\text { Yield } \\
\text { tons } / \text { ha }\end{array}$} \\
\hline & & & & First spray & Second spray & Third spray & & \\
\hline $\mathrm{T} 1$ & $\begin{array}{l}\text { Impression (Tricyclazole } \\
45 \%+\text { Hexaconazole } 10 \% \mathrm{WG} \text { ) }\end{array}$ & 0.75 & $\begin{array}{l}0.92 * \\
(5.49)\end{array}$ & $\begin{array}{c}4.39 \\
(12.08) \\
\end{array}$ & $\begin{array}{l}10.17 \\
(18.6) \\
\end{array}$ & $\begin{array}{c}14.37 \\
(22.28) \\
\end{array}$ & 60.54 & $\begin{array}{c}4.39 \\
(12.08) \\
\end{array}$ \\
\hline $\mathrm{T} 2$ & $\begin{array}{l}\text { Impression (Tricyclazole } \\
45 \%+\text { Hexaconazole } 10 \% \mathrm{WG} \text { ) }\end{array}$ & 1.00 & $\begin{array}{c}0.84 \\
(5.05) \\
\end{array}$ & $\begin{array}{c}4.31 \\
(11.98) \\
\end{array}$ & $\begin{array}{c}9.60 \\
(18.05) \\
\end{array}$ & $\begin{array}{c}12.87 \\
(21.02) \\
\end{array}$ & 64.66 & $\begin{array}{c}5.15 \\
(13.11) \\
\end{array}$ \\
\hline $\mathrm{T} 3$ & $\begin{array}{l}\text { Impression (Tricyclazole } \\
45 \%+\text { Hexaconazole } 10 \% \mathrm{WG} \text { ) }\end{array}$ & 1.25 & $\begin{array}{c}1.01 \\
(5.61)\end{array}$ & $\begin{array}{l}2.25 \\
(8.61) \\
\end{array}$ & $\begin{array}{c}6.25 \\
(14.47) \\
\end{array}$ & $\begin{array}{c}7.17 \\
(15.53) \\
\end{array}$ & 80.32 & $\begin{array}{c}5.25 \\
(13.25)\end{array}$ \\
\hline $\mathrm{T} 4$ & Hexaconazole $5 \%$ EC & 2.00 & $\begin{array}{c}0.84 \\
(5.18) \\
\end{array}$ & $\begin{array}{c}4.25 \\
(11.89)\end{array}$ & $\begin{array}{c}11.17 \\
(19.52)\end{array}$ & $\begin{array}{c}16.74 \\
(24.14) \\
\end{array}$ & 54.05 & $\begin{array}{c}4.14 \\
(11.73) \\
\end{array}$ \\
\hline $\mathrm{T} 5$ & Hexaconazole $5 \%$ EC & 2.50 & $\begin{array}{c}1.09 \\
(5.92) \\
\end{array}$ & $\begin{array}{c}4.17 \\
(11.78) \\
\end{array}$ & $\begin{array}{c}10.92 \\
(19.29) \\
\end{array}$ & $\begin{array}{c}16.42 \\
(23.90)\end{array}$ & 54.92 & $\begin{array}{c}4.14 \\
(11.73) \\
\end{array}$ \\
\hline $\mathrm{T} 6$ & Tricyclazole $75 \% \mathrm{WP}$ & 0.60 & $\begin{array}{c}0.92 \\
(5.36) \\
\end{array}$ & $\begin{array}{c}5.25 \\
(13.24)\end{array}$ & $\begin{array}{c}13.84 \\
(21.84)\end{array}$ & $\begin{array}{c}19.17 \\
(25.95)\end{array}$ & 47.36 & $\begin{array}{c}3.34 \\
(10.50)\end{array}$ \\
\hline $\mathrm{T} 7$ & Tricyclazole 75\% WP & 0.75 & $\begin{array}{c}1.01 \\
(5.61)\end{array}$ & $\begin{array}{c}5.01 \\
(12.92)\end{array}$ & $\begin{array}{c}13.34 \\
(21.42)\end{array}$ & $\begin{array}{c}18.75 \\
(25.62)\end{array}$ & 48.51 & $\begin{array}{c}3.50 \\
(10.79)\end{array}$ \\
\hline $\mathrm{T} 8$ & Propineb $70 \% \mathrm{WP}$ & 3.00 & $\begin{array}{c}0.84 \\
(5.18) \\
\end{array}$ & $\begin{array}{c}4.25 \\
(11.86) \\
\end{array}$ & $\begin{array}{c}13.50 \\
(21.55) \\
\end{array}$ & $\begin{array}{c}14.75 \\
(22.58) \\
\end{array}$ & 59.49 & $\begin{array}{c}4.01 \\
(11.54)\end{array}$ \\
\hline T9 & $\begin{array}{l}\text { Metiram 55\% + Pyraclostrobin } \\
5 \% \text { WG }\end{array}$ & 3.00 & $\begin{array}{c}0.84 \\
(5.18)\end{array}$ & $\begin{array}{c}3.79 \\
(11.19)\end{array}$ & $\begin{array}{c}8.05 \\
(16.48) \\
\end{array}$ & $\begin{array}{c}11.67 \\
(19.94)\end{array}$ & 67.96 & $\begin{array}{c}4.34 \\
(12.02)\end{array}$ \\
\hline $\mathrm{T} 10$ & Untreated Control & NA & $\begin{array}{c}0.92 \\
(5.36) \\
\end{array}$ & $\begin{array}{c}11.50 \\
(19.81)\end{array}$ & $\begin{array}{c}21.62 \\
(27.71)\end{array}$ & $\begin{array}{c}36.42 \\
(37.12) \\
\end{array}$ & 0.00 & $\begin{array}{c}3.04 \\
(10.03)\end{array}$ \\
\hline & \multicolumn{2}{|l|}{ CD@5\% } & NS & 1.42 & 0.96 & 2.11 & - & 0.77 \\
\hline & \multicolumn{2}{|l|}{ SEM } & NS & 0.47 & 0.32 & 0.71 & - & 0.26 \\
\hline
\end{tabular}


superior in reducing the fruit rot incidence (6.25 PDI) of Pomegranate followed by Metiram 55\% + Pyraclostrobin 5\% WG with PDI of 8.05 . The plots treated with Impression (Tricyclazole 45\%+Hexaconazole 10\%WG)@1.00 gm/L and $0.75 \mathrm{gm} / \mathrm{L}$ recorded PDI of 9.60 and 10.17 , respectively and was followed by Hexaconazole 5\%EC @ $2.50 \mathrm{~mL} / \mathrm{L}$ (10.92 PDI). However, the untreated check recorded highest fruit rot incidence by showing 21.62 PDI.

After the third spray schedule similar trend in the efficacy of treatments was recorded, wherein, the plots treated with Impression (Tricyclazole 45\%+Hexaconazole 10\%WG) @ $1.25 \mathrm{gm} / \mathrm{L}$ recorded PDI of 7.17 and there was 80.32 per cent disease reduction over control and it was followed by Metiram 55\% + Pyraclostrobin 5\% WG with PDI of 11.67. The plots treated with Impression (Tricyclazole 45\%+Hexaconazole 10\%WG)@1.00 gm/liter and 0.75 $\mathrm{gm} / \mathrm{L}$ recorded PDI of 12.87 and 14.37 , respectively and higher disease incidence was observed in untreated plot with PDI of 36.42 .

The efficacy of different treatments during three sprays against fruit rot (Colletotrichum sp.) in Pomegranate during 2019-20 is presented in Table-2. At 10 days after first application, Impression (Tricyclazole 45\%+Hexaconazole 10\%WG)@1.25 gm/Lwas found superior in reducing the fruit rot incidence (2.59 PDI) of Pomegranate followed by Metiram 55\% + Pyraclostrobin 5\% WG with PDI of 3.37. The plots treated with Impression (Tricyclazole 45\%+ Hexaconazole 10\%WG)@1.0 gm/L and Hexaconazole 5\% EC@ $2.5 \mathrm{~mL} / \mathrm{L}$ recorded PDI of 4.54 and 4.65, respectively and were on par with each other. Impression (Tricyclazole 45\%+Hexaconazole 10\%WG)@1.0 gm/L, Propineb 70\% WP@ 3.0 gm/L and Hexaconazole 5\% EC@2mL/L recorded PDI of 4.74, 4.84 and 4.84, respectively. The plots treated with Tricyclazole@ $0.75 \mathrm{gm} / \mathrm{L}$ and $0.60 \mathrm{gm} / \mathrm{L}$ recorded PDI of 5.45 and 5.52, respectively. However, the untreated check recorded highest fruit rot incidence by showing 7.75 PDI.

After second spray the treatment of Impression (Tricyclazole 45\%+Hexaconazole 10\%WG) @ $1.25 \mathrm{gm} / \mathrm{Lwas}$ found superior in reducing the fruit rot incidence (8.24 PDI) of Pomegranate followed by Metiram 55\% + Pyraclostrobin 5\% WG with PDI of 10.10 . The plots treated with and Propineb 70\% WP@3gm/L and Impression (Tricyclazole 45\%+Hexaconazole 10\%WG)@1.00 gm/L recorded PDI of 10.94 and 11.90, respectively and Impression (Tricyclazole 45\%+Hexaconazole 10\%WG) @ 0.75gm/L recorded PDI of 12.41. However, the untreated check recorded highest fruit rot incidence by showing 20.89 PDI.

After the third spray schedule similar trend in the efficacy of treatments was recorded, wherein, the plots treated with Impression (Tricyclazole 45\%+Hexaconazole 10\%WG) @ $1.25 \mathrm{gm} / \mathrm{L}$ recorded PDI of 12.30 and there was 66.43 per cent disease reduction over control and it was followed by Metiram 55\% + Pyraclostrobin 5\% WG with PDI of 15.64. The plots treated with Impression (Tricyclazole 45\%+Hexaconazole 10\%WG) @ 1.00 gm/L recorded PDI of 17.80 and higher disease incidence was observed in untreated plot with PDI of 36.65.

Table 2: Bio-efficacy of (Tricyclazole 45\%+Hexaconazole 10\% WG) against Fruit rot (Colletotrichum sp.) disease of Pomegranate during 2019-2020

\begin{tabular}{|c|c|c|c|c|c|c|c|c|}
\hline \multirow{2}{*}{$\begin{array}{l}\text { Tr. } \\
\text { No. }\end{array}$} & \multirow{2}{*}{ Treatments } & \multirow{2}{*}{$\begin{array}{c}\text { Dose } \\
\text { Formulation } \\
(\mathrm{g} / \mathrm{ha})\end{array}$} & \multirow{2}{*}{$\begin{array}{c}\text { Before } \\
\text { spray }\end{array}$} & \multicolumn{3}{|c|}{ PDI 10 days after } & \multirow{2}{*}{$\begin{array}{l}\text { \% Reduction } \\
\text { over control } \\
\text { after } 3^{\text {rd }} \text { spray }\end{array}$} & \multirow{2}{*}{$\begin{array}{c}\text { Yield } \\
\text { tons/ha }\end{array}$} \\
\hline & & & & First spray & Second spray & Third spray & & \\
\hline $\mathrm{T} 1$ & $\begin{array}{l}\text { Impression (Tricyclazole } \\
45 \%+\text { Hexaconazole } 10 \% \mathrm{WG} \text { ) }\end{array}$ & 0.75 & $\begin{array}{l}0.84 * \\
(5.18)\end{array}$ & $\begin{array}{c}4.74 \\
(12.57)\end{array}$ & $\begin{array}{c}12.41 \\
(20.61)\end{array}$ & $\begin{array}{c}19.04 \\
(25.87)\end{array}$ & 48.06 & $\begin{array}{c}4.67 \\
(12.46)\end{array}$ \\
\hline $\mathrm{T} 2$ & \begin{tabular}{|l|} 
Impression (Tricyclazole \\
$45 \%+$ Hexaconazole $10 \% \mathrm{WG}$ )
\end{tabular} & 1.0 & $\begin{array}{c}1.00 \\
(5.71)\end{array}$ & $\begin{array}{c}4.54 \\
(12.26)\end{array}$ & $\begin{array}{c}11.90 \\
(20.18)\end{array}$ & $\begin{array}{c}17.80 \\
(24.96)\end{array}$ & 51.43 & $\begin{array}{c}5.59 \\
(13.67) \\
\end{array}$ \\
\hline $\mathrm{T} 3$ & \begin{tabular}{|l|} 
Impression (Tricyclazole \\
$45 \%+$ Hexaconazole $10 \% \mathrm{WG}$ ) \\
\end{tabular} & 1.25 & $\begin{array}{c}1.00 \\
(5.71)\end{array}$ & $\begin{array}{c}2.59 \\
(9.24)\end{array}$ & $\begin{array}{c}8.24 \\
(16.68) \\
\end{array}$ & $\begin{array}{c}12.30 \\
(20.53)\end{array}$ & 66.43 & $\begin{array}{c}5.64 \\
(13.73) \\
\end{array}$ \\
\hline $\mathrm{T} 4$ & Hexaconazole 5\% EC & 2.0 & $\begin{array}{c}1.00 \\
(5.71)\end{array}$ & $\begin{array}{c}4.84 \\
(12.69) \\
\end{array}$ & $\begin{array}{c}13.34 \\
(21.42)\end{array}$ & $\begin{array}{c}18.84 \\
(25.72)\end{array}$ & 48.61 & $\begin{array}{c}4.44 \\
(12.16) \\
\end{array}$ \\
\hline T5 & Hexaconazole 5\% EC & 2.5 & $\begin{array}{c}0.92 \\
(5.49)\end{array}$ & $\begin{array}{c}4.65 \\
(12.44) \\
\end{array}$ & $\begin{array}{c}13.12 \\
(21.24)\end{array}$ & $\begin{array}{l}18.40 \\
(25.41)\end{array}$ & 49.79 & $\begin{array}{c}4.47 \\
(12.20)\end{array}$ \\
\hline T6 & Tricyclazole $75 \%$ WP & 0.6 & $\begin{array}{c}0.84 \\
(5.18)\end{array}$ & $\begin{array}{c}5.52 \\
(13.59) \\
\end{array}$ & $\begin{array}{c}16.77 \\
(24.17)\end{array}$ & $\begin{array}{c}24.24 \\
(29.49)\end{array}$ & 33.87 & $\begin{array}{c}3.50 \\
(10.75) \\
\end{array}$ \\
\hline $\mathrm{T} 7$ & Tricyclazole $75 \% \mathrm{WP}$ & 0.75 & $\begin{array}{c}0.84 \\
(5.18) \\
\end{array}$ & $\begin{array}{c}5.45 \\
(13.50) \\
\end{array}$ & $\begin{array}{l}16.10 \\
(23.64)\end{array}$ & $\begin{array}{c}23.34 \\
(28.89) \\
\end{array}$ & 36.33 & $\begin{array}{c}3.67 \\
(11.04) \\
\end{array}$ \\
\hline $\mathrm{T} 8$ & Propineb $70 \% \mathrm{WP}$ & 3.0 & $\begin{array}{c}0.84 \\
(5.18) \\
\end{array}$ & $\begin{array}{c}4.84 \\
(12.69) \\
\end{array}$ & $\begin{array}{c}10.94 \\
(19.31) \\
\end{array}$ & $\begin{array}{c}17.24 \\
(24.53) \\
\end{array}$ & 52.97 & $\begin{array}{c}4.17 \\
(11.78) \\
\end{array}$ \\
\hline T9 & $\begin{array}{l}\text { Metiram 55\% + Pyraclostrobin } \\
5 \% \text { WG }\end{array}$ & 3.0 & $\begin{array}{c}0.84 \\
(5.18) \\
\end{array}$ & $\begin{array}{c}3.37 \\
(10.57) \\
\end{array}$ & $\begin{array}{c}10.10 \\
(18.53)\end{array}$ & $\begin{array}{c}15.64 \\
(23.29) \\
\end{array}$ & 57.34 & $\begin{array}{c}4.50 \\
(12.24) \\
\end{array}$ \\
\hline \multirow[t]{3}{*}{$\mathrm{T} 10$} & Untreated Control & NA & $\begin{array}{c}1.01 \\
(5.61)\end{array}$ & $\begin{array}{c}7.75 \\
(16.16) \\
\end{array}$ & $\begin{array}{c}20.89 \\
(27.18)\end{array}$ & $\begin{array}{c}36.65 \\
(37.26) \\
\end{array}$ & 0.00 & $\begin{array}{c}3.20 \\
(10.30)\end{array}$ \\
\hline & \multicolumn{2}{|l|}{$\mathrm{CD} @ 5 \%$} & NS & 1.27 & 1.21 & 1.14 & - & 0.96 \\
\hline & \multicolumn{2}{|l|}{ SEM } & NS & 0.42 & 0.41 & 0.38 & - & 0.32 \\
\hline
\end{tabular}


Efficacy of Tricyclazole $45 \%+$ Hexaconazole $10 \%$ WG against Leaf spot (Alternaria and Cercospora) disease of Pomegranate during 2018-19: The efficacy of different treatments during three sprays against Leaf spot (Alternaria and Cercospora) in Pomegranate during 2018-19 is presented in Table-3. Before the spray, the infestation of leaf spot (Alternaria and Cercospora) was uniform in all the treatments and PDI ranged between 0.84 to 1.09 . At 10 days after first application, Impression (Tricyclazole 45\%+ Hexaconazole 10\%WG) @ 1.25 gm/L and 1.0 gm/Lwere found superior in reducing the fruit rot incidence with 2.35 and 2.50 PDI where these two treatments were on par with other and which was followed by (Tricyclazole 45\%+Hexaconazole 10\%WG)@ 0.75 gm/L (3.82 PDI). The plots treated with Metiram 55\% + Pyraclostrobin 5\% WG recorded PDI of 3.87. Hexaconazole 5\% EC @ $2.5 \mathrm{~mL} / \mathrm{L}$ and $2 \mathrm{~mL} / \mathrm{L}$ recorded PDI of 4.25 and 4.34, respectively. Propineb 70\% WP@3.0 gm/L recorded PDI of 4.42. However, the untreated check recorded highest fruit rot incidence by showing 12.09 PDI.

After second spray the treatment of Impression (Tricyclazole 45\%+Hexaconazole 10\%WG)@1.25 gm/L and $1.0 \mathrm{gm} / \mathrm{L}$ were found superior in reducing the fruit rot incidence with 6.77 and 7.07 PDI, respectively. The plots treated with Metiram 55\% + Pyraclostrobin 5\% WG recorded PDI of 8.15. The plots treated with Impression (Tricyclazole 45\%+Hexaconazole 10\%WG) @ $0.75 \mathrm{gm} / \mathrm{L}$ recorded PDI of 9.64. Hexaconazole 5\%EC @ $2.50 \mathrm{~mL} / \mathrm{L}$ and $2.0 \mathrm{~mL} / \mathrm{L}$ recorded PDI of 11.04 and 11.27 , respectively where these two treatments were on par with each other. The plots treated with Tricyclazole 75\%WP @ $0.75 \mathrm{gm} / \mathrm{L}$ and $0.6 \mathrm{gm} / \mathrm{L}$ recorded PDI of 13.44 and 13.94 which were on par with each other. However, the untreated check recorded highest fruit rot incidence by showing 22.47 PDI.

After the third spray schedule similar trend in the efficacy of treatments was recorded, wherein, the plots treated with Impression (Tricyclazole 45\%+Hexaconazole 10\%WG) @ $1.25 \mathrm{gm} / \mathrm{L}$ and $1.0 \mathrm{gm} / \mathrm{L}$ recorded PDI of 9.64 and 9.97 which were superior over other treatments and there was 74.02 per cent disease reduction over control and it was followed by Metiram 55\% + Pyraclostrobin 5\% WG with PDI of 11.79 . The plots treated with Hexaconazole @2.5 mL/L and 2.0 $\mathrm{mL} / \mathrm{L}$ recorded disease incidence of 11.04 and 11.27, respectively and higher disease incidence was observed in untreated plot with PDI of 37.09

The efficacy of different treatments during three sprays against Leaf spot (Alternaria and Cercospora) in Pomegranate during 2019-20 is presented in Table-4. Before the spray, the infestation of leaf spot (Alternaria and Cercospora) was uniform in all the treatments and PDI ranged between 0.84 to 1.09. At 10 days after first application, Impression (Tricyclazole 45\%+Hexaconazole 10\%WG) @ $1.25 \mathrm{gm} / \mathrm{L}$ and $1.0 \mathrm{gm} / \mathrm{L}$ were found superior in reducing the fruit rot incidence with 2.92 and 3.00 PDI where these two treatments were at par with other and which was followed by Metiram 55\% + Pyraclostrobin 5\% WG which recorded PDI of 3.70. Impression(Tricyclazole 45\%+Hexaconazole 10\%WG) @ 0.75 gm/L recorded PDI of 4.47. The plots treated with Hexaconazole 5\% EC @ 2.5 mL/L and $2 \mathrm{~mL} / \mathrm{L}$ recorded PDI of 4.99 and 5.17, respectively. However, the untreated check recorded highest fruit rot incidence by showing 8.09 PDI.

After second spray the treatment of Impression (Tricyclazole 45\%+Hexaconazole 10\%WG) @ $1.25 \mathrm{gm} / \mathrm{L}$ and $1.0 \mathrm{gm} / \mathrm{L}$ were found to be superior in reducing the fruit rot incidence with 8.57 and 8.70 PDI, respectively which were on par with

Table 3: Bio-efficacy of (Tricyclazole 45\%+Hexaconazole 10\%WG) against Leaf spot (Alternaria and Cercospora) disease of Pomegranate during 2018-19

\begin{tabular}{|c|c|c|c|c|c|c|c|c|}
\hline \multirow{2}{*}{$\begin{array}{l}\text { Tr. } \\
\text { No. }\end{array}$} & \multirow{2}{*}{ Treatments } & \multirow{2}{*}{$\begin{array}{c}\text { Dose } \\
\text { Formulation } \\
(\mathrm{g} / \mathrm{ha})\end{array}$} & \multirow{2}{*}{$\begin{array}{l}\text { Before } \\
\text { spray }\end{array}$} & \multicolumn{3}{|c|}{ PDI 10 days after } & \multirow{2}{*}{$\begin{array}{l}\text { \% Reduction } \\
\text { over control } \\
\text { after } 3^{\text {rd }} \text { spray }\end{array}$} & \multirow{2}{*}{$\begin{array}{c}\text { Yield } \\
\text { tons/ha }\end{array}$} \\
\hline & & & & First spray & Second spray & Third spray & & \\
\hline $\mathrm{T} 1$ & $\begin{array}{l}\text { Impression (Tricyclazole } \\
45 \%+\text { Hexaconazole } 10 \% \mathrm{WG} \text { ) }\end{array}$ & 0.75 & $\begin{array}{c}0.92 \\
(5.49) \\
\end{array}$ & $\begin{array}{c}3.82 \\
(11.27) \\
\end{array}$ & $\begin{array}{c}9.64 \\
(18.09) \\
\end{array}$ & $\begin{array}{c}15.30 \\
(23.03) \\
\end{array}$ & 58.74 & $\begin{array}{c}4.39 \\
(12.08)\end{array}$ \\
\hline $\mathrm{T} 2$ & $\begin{array}{l}\text { Impression (Tricyclazole } \\
45 \%+\text { Hexaconazole } 10 \% \mathrm{WG} \text { ) }\end{array}$ & 1.0 & $\begin{array}{c}0.84 \\
(5.05) \\
\end{array}$ & $\begin{array}{c}2.50 \\
(9.10) \\
\end{array}$ & $\begin{array}{c}7.07 \\
(15.42) \\
\end{array}$ & $\begin{array}{c}9.97 \\
(18.36) \\
\end{array}$ & 73.12 & $\begin{array}{c}5.15 \\
(13.11) \\
\end{array}$ \\
\hline $\mathrm{T} 3$ & $\begin{array}{l}\text { Impression (Tricyclazole } \\
45 \%+\text { Hexaconazole } 10 \% \mathrm{WG} \text { ) }\end{array}$ & 1.25 & $\begin{array}{c}1.00 \\
(5.61)\end{array}$ & $\begin{array}{l}2.35 \\
(8.8)\end{array}$ & $\begin{array}{c}6.77 \\
(15.07) \\
\end{array}$ & $\begin{array}{c}9.64 \\
(18.09) \\
\end{array}$ & 74.02 & $\begin{array}{c}5.25 \\
(13.25)\end{array}$ \\
\hline $\mathrm{T} 4$ & Hexaconazole $5 \%$ EC & 2.0 & $\begin{array}{c}0.84 \\
(5.18) \\
\end{array}$ & $\begin{array}{c}4.34 \\
(12.01) \\
\end{array}$ & $\begin{array}{c}11.27 \\
(19.62) \\
\end{array}$ & $\begin{array}{c}16.84 \\
(24.22) \\
\end{array}$ & 54.60 & $\begin{array}{c}4.14 \\
(11.73) \\
\end{array}$ \\
\hline $\mathrm{T} 5$ & Hexaconazole $5 \%$ EC & 2.5 & $\begin{array}{c}1.09 \\
(5.92) \\
\end{array}$ & $\begin{array}{c}4.25 \\
(11.90)\end{array}$ & $\begin{array}{c}11.04 \\
(19.40)\end{array}$ & $\begin{array}{c}16.54 \\
(23.99)\end{array}$ & 55.41 & $\begin{array}{c}4.14 \\
(11.73)\end{array}$ \\
\hline T6 & Tricyclazole $75 \%$ WP & 0.6 & $\begin{array}{c}0.92 \\
(5.36) \\
\end{array}$ & $\begin{array}{c}5.34 \\
(13.35) \\
\end{array}$ & $\begin{array}{c}13.94 \\
(21.92) \\
\end{array}$ & $\begin{array}{c}19.27 \\
(26.02)\end{array}$ & 48.04 & $\begin{array}{c}3.34 \\
(10.5) \\
\end{array}$ \\
\hline $\mathrm{T} 7$ & Tricyclazole $75 \%$ WP & 0.75 & $\begin{array}{c}1.01 \\
(5.61) \\
\end{array}$ & $\begin{array}{c}5.17 \\
(13.13) \\
\end{array}$ & $\begin{array}{c}13.44 \\
(21.50) \\
\end{array}$ & $\begin{array}{c}18.85 \\
(25.69) \\
\end{array}$ & 49.16 & $\begin{array}{c}3.50 \\
(10.79) \\
\end{array}$ \\
\hline $\mathrm{T} 8$ & Propineb $70 \% \mathrm{WP}$ & 3.0 & $\begin{array}{c}0.84 \\
(5.18) \\
\end{array}$ & $\begin{array}{c}4.42 \\
(12.12) \\
\end{array}$ & $\begin{array}{c}13.60 \\
(21.63) \\
\end{array}$ & $\begin{array}{c}14.85 \\
(22.66) \\
\end{array}$ & 59.95 & $\begin{array}{c}4.00 \\
(11.54) \\
\end{array}$ \\
\hline T9 & $\begin{array}{l}\text { Metiram 55\% + Pyraclostrobin } \\
5 \% \text { WG }\end{array}$ & 3.0 & $\begin{array}{c}0.84 \\
(5.18) \\
\end{array}$ & $\begin{array}{c}3.87 \\
(11.32) \\
\end{array}$ & $\begin{array}{c}8.15 \\
(16.59) \\
\end{array}$ & $\begin{array}{c}11.79 \\
(20.05) \\
\end{array}$ & 68.22 & $\begin{array}{c}4.34 \\
(12.02)\end{array}$ \\
\hline \multirow[t]{3}{*}{$\mathrm{T} 10$} & Untreated Control & NA & $\begin{array}{c}0.92 \\
(5.36) \\
\end{array}$ & $\begin{array}{c}12.09 \\
(20.34)\end{array}$ & $\begin{array}{c}22.47 \\
(28.30)\end{array}$ & $\begin{array}{c}37.09 \\
(37.52) \\
\end{array}$ & 0.00 & $\begin{array}{c}3.04 \\
(10.03)\end{array}$ \\
\hline & \multicolumn{2}{|l|}{$\mathrm{CD} @ 5 \%$} & $\mathrm{NS}$ & 1.07 & 0.88 & 2.30 & - & 0.77 \\
\hline & \multicolumn{2}{|l|}{ SEM } & NS & 0.36 & 0.29 & 0.37 & - & 0.26 \\
\hline
\end{tabular}


Table 4. Bio-efficacy of (Tricyclazole 45\%+Hexaconazole 10\%WG) against Leaf spot (Alternaria and Cercospora) disease of Pomegranate during 2019-2020

\begin{tabular}{|c|c|c|c|c|c|c|c|c|}
\hline \multirow{2}{*}{$\begin{array}{l}\text { Tr. } \\
\text { No. }\end{array}$} & \multirow[b]{2}{*}{ Treatments } & \multirow{2}{*}{$\begin{array}{c}\text { Dose } \\
\text { Formulation } \\
\text { (g/ha) }\end{array}$} & \multirow{2}{*}{$\begin{array}{l}\text { Before } \\
\text { spray }\end{array}$} & \multicolumn{3}{|c|}{ PDI 10 days after } & \multirow{2}{*}{$\begin{array}{l}\% \text { Reduction } \\
\text { over control } \\
\text { after } 3^{\text {rd }} \text { spray }\end{array}$} & \multirow[b]{2}{*}{ Yield tons/ha } \\
\hline & & & & First spray & Second spray & Third spray & & \\
\hline $\mathrm{T} 1$ & $\begin{array}{l}\text { Impression (Tricyclazole } \\
45 \%+\text { Hexaconazole } 10 \% \mathrm{WG} \text { ) }\end{array}$ & 0.75 & $\begin{array}{l}0.84 * \\
(5.18)\end{array}$ & $\begin{array}{c}4.47 \\
(12.18) \\
\end{array}$ & $\begin{array}{l}11.65 \\
(19.96) \\
\end{array}$ & $\begin{array}{c}16.40 \\
(23.89) \\
\end{array}$ & 55.65 & $\begin{array}{c}4.67 \\
(12.46) \\
\end{array}$ \\
\hline $\mathrm{T} 2$ & $\begin{array}{l}\text { Impression (Tricyclazole } \\
45 \%+\text { Hexaconazole } 10 \% \mathrm{WG} \text { ) }\end{array}$ & 1.0 & $\begin{array}{c}1.00 \\
(5.71) \\
\end{array}$ & $\begin{array}{l}3.00 \\
(9.95) \\
\end{array}$ & $\begin{array}{c}8.70 \\
(17.15) \\
\end{array}$ & $\begin{array}{c}12.27 \\
(20.46) \\
\end{array}$ & 66.83 & $\begin{array}{c}5.59 \\
(13.67) \\
\end{array}$ \\
\hline $\mathrm{T} 3$ & $\begin{array}{l}\text { Impression (Tricyclazole } \\
45 \%+\text { Hexaconazole } 10 \% \mathrm{WG} \text { ) }\end{array}$ & 1.25 & $\begin{array}{c}1.00 \\
(5.71)\end{array}$ & $\begin{array}{c}2.92 \\
(9.79)\end{array}$ & $\begin{array}{c}8.57 \\
(17.02) \\
\end{array}$ & $\begin{array}{c}12.00 \\
(20.27)\end{array}$ & 67.55 & $\begin{array}{c}5.64 \\
(13.73) \\
\end{array}$ \\
\hline $\mathrm{T} 4$ & Hexaconazole 5\% EC & 2.0 & $\begin{array}{c}1.00 \\
(5.71)\end{array}$ & $\begin{array}{c}5.17 \\
(13.09)\end{array}$ & $\begin{array}{c}13.67 \\
(21.70)\end{array}$ & $\begin{array}{c}18.92 \\
(25.78)\end{array}$ & 48.85 & $\begin{array}{c}4.44 \\
(12.16) \\
\end{array}$ \\
\hline T5 & Hexaconazole $5 \%$ EC & 2.5 & $\begin{array}{c}0.92 \\
(5.49)\end{array}$ & $\begin{array}{c}4.99 \\
(12.88) \\
\end{array}$ & $\begin{array}{c}13.45 \\
(21.52) \\
\end{array}$ & $\begin{array}{c}18.49 \\
(25.47) \\
\end{array}$ & 50.02 & $\begin{array}{c}4.47 \\
(12.20) \\
\end{array}$ \\
\hline T6 & Tricyclazole 75\% WP & 0.6 & $\begin{array}{c}0.84 \\
(5.18)\end{array}$ & $\begin{array}{c}5.85 \\
(13.98) \\
\end{array}$ & $\begin{array}{c}17.10 \\
(24.43)\end{array}$ & $\begin{array}{c}24.32 \\
(29.55)\end{array}$ & 34.25 & $\begin{array}{c}3.50 \\
(10.75)\end{array}$ \\
\hline $\mathrm{T} 7$ & Tricyclazole 75\% WP & 0.75 & $\begin{array}{c}0.84 \\
(5.18) \\
\end{array}$ & $\begin{array}{c}5.79 \\
(13.90) \\
\end{array}$ & $\begin{array}{c}16.44 \\
(23.90) \\
\end{array}$ & $\begin{array}{c}23.42 \\
(28.94) \\
\end{array}$ & 36.68 & $\begin{array}{c}3.67 \\
(11.04) \\
\end{array}$ \\
\hline $\mathrm{T} 8$ & Propineb $70 \% \mathrm{WP}$ & 3.0 & $\begin{array}{c}0.84 \\
(5.18)\end{array}$ & $\begin{array}{c}5.17 \\
(13.14)\end{array}$ & $\begin{array}{c}11.27 \\
(19.61)\end{array}$ & $\begin{array}{c}17.35 \\
(24.62)\end{array}$ & 53.08 & $\begin{array}{c}4.17 \\
(11.78)\end{array}$ \\
\hline T9 & $\begin{array}{l}\text { Metiram 55\%+ Pyraclostrobin } \\
5 \% \text { WG }\end{array}$ & 3.0 & $\begin{array}{c}0.84 \\
(5.18) \\
\end{array}$ & $\begin{array}{c}3.70 \\
(11.06) \\
\end{array}$ & $\begin{array}{c}10.44 \\
(18.84) \\
\end{array}$ & $\begin{array}{c}15.72 \\
(23.36) \\
\end{array}$ & 57.50 & $\begin{array}{c}4.50 \\
(12.24) \\
\end{array}$ \\
\hline \multirow[t]{3}{*}{$\mathrm{T} 10$} & Untreated Control & NA & $\begin{array}{c}1.00 \\
(5.61) \\
\end{array}$ & $\begin{array}{c}8.09 \\
(16.52) \\
\end{array}$ & $\begin{array}{c}21.22 \\
(27.43) \\
\end{array}$ & $\begin{array}{c}36.99 \\
(37.46) \\
\end{array}$ & 0.00 & $\begin{array}{c}3.20 \\
(10.30) \\
\end{array}$ \\
\hline & \multicolumn{2}{|l|}{ CD@5\% } & NS & 1.77 & 1.24 & 1.46 & - & 0.96 \\
\hline & \multicolumn{2}{|l|}{ SEM } & NS & 0.59 & 0.41 & 0.49 & - & 0.32 \\
\hline
\end{tabular}

each other. The plots treated with Metiram 55\% + Pyraclostrobin 5\% WG recorded PDI of 10.44 . The plots treated with Impression (Tricyclazole 45\%+Hexaconazole $10 \% \mathrm{WG}$ @ 0.75gm $/ \mathrm{L}$ recorded PDI of 11.65 . The Plots treated with Propineb 70\% WP recorded PDI of 11.27 . Hexaconazole 5\%EC @ 2.50 mL/L and $2.0 \mathrm{~mL} / \mathrm{L}$ recorded PDI of 13.45 and 13.67 , respectively where these two treatments were on par with each other. The plots treated with Tricyclazole 75\%WP@0.75gm/L and $0.6 \mathrm{gm} / \mathrm{L}$ recorded PDI of 16.44 and 17.10 which were on par with each other. However, the untreated check recorded highest fruit rot incidence by showing 21.22 PDI.

After the third spray schedule similar trend in the efficacy of treatments was recorded, wherein, the plots treated with Impression (Tricyclazole 45\%+Hexaconazole 10\%WG) @ $1.25 \mathrm{gm} / \mathrm{L}$ and $1.0 \mathrm{gm} / \mathrm{L}$ recorded PDI of 12.00 and 12.27 which were superior over other treatments and there was 67.55 per cent disease reduction over control and it was followed by Metiram 55\% + Pyraclostrobin 5\% WG with PDI of 15.72. The plots treated with Hexaconazole @2.5 mL/L and $2.0 \mathrm{~mL} / \mathrm{L}$ recorded disease incidence of 18.49 and 18.92 , respectively and higher disease incidence was observed in untreated plot with PDI of 36.99.

Fruit yield: The efficacy of different treatments during three applications resulted in difference in Pomegranate yield per plant during 2018-19 which is presented in Table-1. Application of Impression (Tricyclazole 45\%+Hexaconazole $10 \% \mathrm{WG}) @ 1.25 \mathrm{gm} / \mathrm{L}$ and $1.0 \mathrm{gm} / \mathrm{L}$ resulted in higher pomegranate fruit yield of 5.25 tons/ha and 5.15 tons/ha which were at par with each other and these treatments remained statistically superior over all the treatment. The plots treated with Impression (Tricyclazole 45\%+ Hexaconazole 10\%WG)@0.75 gm/L and Metiram 55\% + Pyraclostrobin 5\% WG recorded a yield of 4.39 tons/ha and 4.34 tons/ha, respectively. However, lowest yield was recorded in the untreated check (3.04 tons/ha).

The efficacy of different treatments during three applications resulted in difference in Pomegranate yield per hectare during 2019-20 is presented in Table-2. Application of Impression (Tricyclazole 45\%+Hexaconazole 10\%WG)@ 1.25 gm/L and $1.0 \mathrm{gm} / \mathrm{L}$ also recorded in higher pomegranate fruit yield of 5.64 tons/ha and 5.59 tons/ha which were at par with each other and these treatments remained statistically superior over all the treatment. The plots treated with Impression (Tricyclazole 45\%+Hexaconazole 10\%WG)@0.75 gm/L and Metiram 55\% + Pyraclostrobin 5\% WG recorded a yield of 4.67 tons/ha and 4.50 tons/ha, respectively. However, lowest yield was recorded in the untreated check (3.20 tons/ha).

Phytotoxic effect of Impression (Tricyclazole $45 \%+$ Hexaconazole 10\%WG) on Pomegranate crop during 2018-19 and 2019-20: Application of Impression (Tricyclazole 45\%+Hexaconazole 10\%WG)@5.0gm/L, 2.5 $\mathrm{gm} / \mathrm{L}, 1.25 \mathrm{gm} / \mathrm{L}$ and $1.0 \mathrm{gm} / \mathrm{L}$ dose rates and other tested chemicals when examined for its phytotoxic effects. No phytotoxicity symptoms like leaf injury, wilting, vein clearing, necrosis, epinasty and hyponasty were detected after treatments on pomegranate crop (Table-5).

Efficacy of tricyclazole, hexaconazole and strobilurins against blast has also been reported earlier (Ram Singh et al., 2004; 2010; Srinivas Prasad et al., 2011; Upmanyu and Rana, 2012). Magar et al., (2015) reported that Tricyclazole 22\% + Hexaconazole 3\% SC was found to be effective in the 
Table 5: Evaluation of Tricyclazole 45\%+Hexaconazole 10\%WG for Phytotoxicity on Pomegranate during 2018-19 and 2019-2020

\begin{tabular}{|c|c|c|c|c|c|c|c|c|c|c|c|c|c|c|c|c|c|c|c|c|c|c|c|}
\hline \multirow{3}{*}{$\begin{array}{l}\text { Tr. } \\
\text { No. }\end{array}$} & \multirow{3}{*}{ Treatments } & \multirow{3}{*}{$\begin{array}{l}\text { Dosage } \\
\text { (gm /lt) }\end{array}$} & \multicolumn{21}{|c|}{ Phytotoxicity observations at $0,1,3,5,7 \& 10$ days after application (Scale: $0-9$ ) } \\
\hline & & & \multicolumn{3}{|c|}{$\begin{array}{c}\text { Leaf tip } \\
\text { injury }\end{array}$} & \multicolumn{3}{|c|}{ Wilting } & \multicolumn{3}{|c|}{$\begin{array}{c}\text { Vein } \\
\text { clearing }\end{array}$} & \multicolumn{3}{|c|}{ Necrosis } & \multicolumn{3}{|c|}{ Epinasty } & \multicolumn{3}{|c|}{ Yellowing } & \multicolumn{3}{|c|}{ Hyponasty } \\
\hline & & & $R_{1}$ & $\mathbf{R}_{2}$ & $\mathbf{R}_{3}$ & $\mathbf{R}_{1}$ & $\mathbf{R}_{2}$ & $\mathbf{R}_{3}$ & $R_{1}$ & $\mathbf{R}_{2}$ & $\mathbf{R}_{3}$ & $\mathbf{R}_{1}$ & $\mathbf{R}_{\mathbf{2}}$ & $\mathbf{R}_{3}$ & $\mathbf{R}_{1}$ & $\mathbf{R}_{\mathbf{2}}$ & $\mathbf{R}_{\mathbf{3}}$ & $\mathbf{R}_{1}$ & $\mathbf{R}_{2}$ & $\mathbf{R}_{3}$ & $\mathbf{R}_{1}$ & $\mathbf{R}_{2}$ & $\mathbf{R}_{\mathbf{3}}$ \\
\hline $\mathrm{T} 2$ & \begin{tabular}{|l|} 
Impression (Tricyclazole \\
$45 \%+$ Hexaconazole $10 \% \mathrm{WG}$ )
\end{tabular} & $1.0 \mathrm{~g}$ & $00^{*}$ & 00 & 00 & 00 & 00 & 00 & 00 & 00 & 00 & 00 & 00 & 00 & 00 & 00 & 00 & 00 & 00 & 00 & 00 & 00 & 00 \\
\hline $\mathrm{T} 3$ & $\begin{array}{l}\text { Impression (Tricyclazole } \\
45 \%+\text { Hexaconazole } 10 \% \mathrm{WG} \text { ) }\end{array}$ & $1.25 \mathrm{~g}$ & 00 & 00 & 00 & 00 & 00 & 00 & 00 & 00 & 00 & 00 & 00 & 00 & 00 & 00 & 00 & 00 & 00 & 00 & 00 & 00 & 00 \\
\hline $\mathrm{T} 10$ & Untreated check & NA & 00 & 00 & 00 & 00 & 00 & 00 & 00 & 00 & 00 & 00 & 00 & 00 & 00 & 00 & 00 & 00 & 00 & 00 & 00 & 00 & 00 \\
\hline $\mathrm{T} 11$ & $\begin{array}{l}\text { Impression (Tricyclazole } \\
45 \%+\text { Hexaconazole } 10 \mathrm{WG} \text { ) }\end{array}$ & $2.5 \mathrm{~g}$ & 00 & 00 & 00 & 00 & 00 & 00 & 00 & 00 & 00 & 00 & 00 & 00 & 00 & 00 & 00 & 00 & 00 & 00 & 00 & 00 & 00 \\
\hline $\mathrm{T} 12$ & $\begin{array}{l}\text { Impression (Tricyclazole } \\
45 \%+\text { Hexaconazole } 10 \mathrm{WG} \text { ) }\end{array}$ & $5.0 \mathrm{~g}$ & 00 & 00 & 00 & 00 & 00 & 00 & 00 & 00 & 00 & 00 & 00 & 00 & 00 & 00 & 00 & 00 & 00 & 00 & 00 & 00 & 00 \\
\hline
\end{tabular}

management of leaf blast and neck blast incidence and highest percentage disease control. Similar findings about the efficacy of Tricyclazole + Hexaconazole were also reported by Sachin and Sandeep (2016). Chethana (2018) reported that Tricyclazole $45 \%+$ hexaconazole $10 \%$ WG at higher concentration did not show any symptoms of phyto-toxicity till 15 days after application and present results were in agreement with these studies. Efficacy of these two new combination products is due to tricyclazole as one of the constituents (Tricyclazole + Hexaconazole). Use of tricyclazole, a melanin biosynthesis inhibitor, has been advocated by Yamaguchi (2004) as this is an environmentally safe fungicide and is less likely to lead to resistance development in the pathogen. Hexaconazole is a broadspectrum systemic fungicide. The mode of action is that it is a sterol demethylation inhibitor that is reported to prevent the development of the fungus by inhibiting cell membrane ergosterol biosynthesis (Bisht et al., 2020). The combination of tricyclazole with chemicals of different modes of action like mancozeb and hexaconazole may further reduce the risk of resistance development in the pathogen besides being more effective than tricyclazole alone.

\section{CONCLUSION}

Impression (Tricyclazole 45\%+Hexaconazole 10\%WG) @ $1.25 \mathrm{gm} / \mathrm{L}$ and $1.0 \mathrm{gm} / \mathrm{L}$ was found to be an optimal dose which help in significantly reducing the Pomegranate diseases like Cercospora and Alternaria leaf spot and Anthracnose or fruit spot.

\section{ACKNOWLEDGEMENT}

The senior author is grateful to $\mathrm{M} / \mathrm{s}$ Indofil Industries Ltd, Mumbai for providing financial assistance to conduct the study.

\section{REFERENCE}

Anonymous, 2004. Improved Cultivation Practices for Horticulture Crops. University of Agricultural Sciences, Dharwad, India, p. 470.

Chethana, B. S. 2018.A New Combination Fungicide for the Management of Sheath Blight and Neck Blast Diseases of Paddy. Int. J. Pure Appl. Biosci. 6(4):651-655.

Bartlett, D. W. Clough, J. M. Godwin, J. R. Hall, A. A. and
Hamer, M. Parr-dobrzanski, B. 2002. The strobilurin fungicides. Pest Manag. Sci. 58: 649-662.

Bisht, T. S., Lakshmi, R., Dinesh, C., Naithani, Prasad, R. K. and Ankit, T. 2018.Bio-efficacy evaluation of Chlorathalonil 40\% w/w + Difenconazole 4\% w/w SC against apple diseases. Int. J. Che. Stu. 8 (1): 398405.

De, R. K. and Mandal, R. K. 2007. Effect of seed treatment with fungicides on foot and stem rot disease caused by Phytophthora parasitica var. sabdariffaein Hibiscus sabdariffa. J. Inter. academicia 11:161165.

Jamadar, M. M. and Patil, D. R. 2007. Bio-efficacy of new formulations against Leaf/Fruit Spot on Pomegranate. Karnataka J. Agric. Sci. 20 (4): $865-$ 866.

Magar, P. B. Acharya, B. and Pandey, B. 2015. Use of chemical fungicides for the management of rice blast (Pyricularia grisea) disease at Jyotinagar, chitwan. Nepal Int. J. Appl. Sci. Biotech. 3(3): 474478.

Nithyameenakshi, S. Jeyaramraja, P. R. and Manian, S.2006, Investigations on phytotoxicity of two new fungicides azoxystrobin and difenoconazole. American J. Pl. Physiol. 1:89-98.

Ram Singh, Sunder, S. and Dodan, D. S. 2004. Evaluation of scented rice genotypes to blast and its management with fungicides. J. Mycol. Pl. Pathol. 34: 280-281.

Ram Singh, Sunder, S.Dodan, D. S.,Ram, L. and Singh, R. 2010. Evaluation of scented rice genotypes and fungicides against blast and compatibility of pesticides used against neck blast, stem borer and leaf folder. Indian Phytopathol. 63: 212-215.

Sachin, U. and Sandeep, M. 2016, Evaluation of new fungicides for the management of neck blast in rice (Oryza sativa L.). Himachal J. Agric. Res. 42 (1): 114-116.

Srinivas Prasad, M., Sheshumadhav, M., Laha, G. S., Ladha Lalshmi, D., Krishnaveni, D., Mangrauthia, S. K., Balachandran, S. M. Sundaram, R. M., 
Arunakranthi, B.,Madhan Mohan, K., Ratnamadhavi, K., Kumar, V. andViraktamath, B. C.2011. Rice blast disease and its management. Technical Bulletin No. 57. Directorate of Rice Research (ICAR), Rajendranagar, Hyderabad. pp 52.

Sundarraj, N.,Nagaraja, S., Venkataramu, M. S. and Jaganath, M. K. 1974. Design and analysis of field experiments. Mysore.

Upmanyu, S. and Rana, S. K. 2012. Effect of fungicides on neck blast incidence and grain yield of rice in midhills of Himachal Pradesh. Pl. Dis. Res. 27 (1): 92-93.

Walter, P. F. 1997. Experimental design theory and application. $3^{\text {rd }}$ Edition, New York.

Wheeler, B. E. J. 1969. An Introduction to Plant Diseases. John Wiley and Sons Ltd. London, p. 301.

Yamaguchi, I. 2004. Overview on the chemical control ofrice blast disease. In: Rice blast: Interaction with rice and control (Ed.: Shinji Kawasaki). Springer Netherlands. pp 1-13. 